\title{
Can a Multi-Epitope Vaccine be a solution for COVID-19 pandemic?
}

\author{
Domina Petric, MD
}

\begin{abstract}
Problems associated with Wuhan spike protein-based vaccines are the emergence of new SARS-CoV-2 strains that are more or less resistant to Wuhan spike protein-based vaccine-induced immunity, and toxicity of the spike protein (S protein alone can damage vascular endothelial cells).

Multi-epitope vaccine against SARS-CoV-2, excluding the spike protein, could potentially be less toxic than Wuhan spike protein-based vaccines with less serious side effects, but still enough immunogenic to elicit protective immune response. Another potential benefit of a multi-epitope COVID-19 vaccine is that, taking into account the fact that most of the new SARS-CoV-2 strains carry significant new mutations in the spike protein and less significant mutations in other structural proteins of the virus, it could provide more potent immune response against new SARSCoV-2 variants.
\end{abstract}

INTRODUCTION

Problems associated with Wuhan spike protein based vaccines, which are BNT162b2 (Pfizer-BioNTech COVID-19 vaccine: mRNA vaccine delivered in a lipid nanoparticle to express a full-length spike protein), mRNA-1273 (Moderna COVID-19 vaccine: messenger RNA vaccine that was one of the first vaccines for SARS-CoV-2 to be produced; it was developed and administered to humans within two months of publication of the SARS-CoV-2 genomic sequence), Ad26.COV2.S (Janssen/Johnson \& Johnson COVID-19 vaccine: this vaccine is based on a replication-incompetent adenovirus 26 vector that expresses a stabilized spike protein), ChAdOx1 nCoV19/AZD1222 (University of Oxford, AstraZeneca, and the Serum Institute of India: this vaccine is based on a replicationincompetent chimpanzee adenovirus vector that expresses the spike protein), NVXCoV2373 (Novavax: this is a recombinant protein nanoparticle vaccine composed of trimeric spike glycoproteins and a potent Matrix-M1 adjuvant), Ad5-based COVID19 vaccine (CanSino Biologics: this vaccine 
is based on a replication-incompetent adenovirus 5 vector that expresses the spike protein), Gam-COVID-Vac/Sputnik V (Gamaleya Institute: this is a vaccine developed in Russia that uses two replicationincompetent adenovirus vectors that express a full-length spike glycoprotein) $)^{1}$, are:

1. The emergence of new SARS-CoV-2 strains (spike protein-based mutations) that are more or less resistant to Wuhan spike proteinbased vaccine-induced immunity.

2. Toxicity of the spike protein.

\section{NEW SARS-CoV-2 STRAINS}

According to World Health Organization ${ }^{2}$ SARS-CoV-2 variant that meets the definition of variant of concern (VOC) is one that has been demonstrated to be associated with one or more of the following chances at a degree of global public health significance:

1. Increase in transmissibility or detrimental change in COVID-19 epidemiology; OR

2. Increase in virulence or change in clinical disease presentation; OR

3. Decrease in effectiveness of public health and social measures or available diagnostics, vaccines, therapeutics.

VOC are:

1. Alpha (B.1.1.7; notable spike (S) amino acid changes under monitoring are $+\mathrm{S}: 484 \mathrm{~K}$ and $+\mathrm{S}$ : 452R; United Kingdom)

2. Beta (B.1.351, B.1.351.2, B.1.351.3; notable spike (S) amino acid change under monitoring is $+\mathrm{S}$ : L18F; South Africa)

3. Gamma (P.1, P.1.1, P.1.2, P.1.4, P.1.6, P.1.7; notable spike (S) amino acid change under monitoring is $+\mathrm{S}$ : 681H; Brazil)

4. Delta (B.1.617.2, AY.1, AY.2, AY.3, AY.3.1; notable spike (S) amino acid change under monitoring is $+\mathrm{S}: 417 \mathrm{~N}$; India)

A SARS-CoV variant of interest (VOI) is one with genetic changes that are predicted or known to affect virus characteristics such as transmissibility, disease severity, immune escape, diagnostic or therapeutic escape; and identified to cause significant community transmission or multiple COVID-19 clusters, in multiple countries with increasing relative prevalence alongside increasing number of cases over time, or other apparent 
epidemiological impacts to suggest an emerging risk to global public health.

VOI are:

1. Eta (B.1.525, multiple countries)

2. Iota (B.1.526, United States of America)

3. Kappa (B.1.617.1, India)

4. Lambda (C.37, Peru)

The Delta Plus variant has a significant number of high-prevalence mutations $(\geq 20 \%$ than in the Delta variant). Signature mutations in Spike (G142D, A222V, and T95I) exist at a more significant percentage in the Delta Plus variant than the Delta variant. Three mutations in Spike (K417N, V70F, and W258L) are exclusively present in the Delta Plus variant. A new mutation was identified in ORF1a (A1146T), which is only present in the Delta Plus variant with $\sim 58 \%$ prevalence. Five key mutations (T95I, A222V, G142D, $\mathrm{R} 158 \mathrm{G}$, and $\mathrm{K} 417 \mathrm{~N}$ ) are significantly more prevalent in the Delta Plus than in the Delta variant. Structural analyses revealed that mutations alter the sidechain conformation to weaken the interactions with antibodies ${ }^{3}$.

A novel variant of concern named CAL.20C (B.1.427/B.1.429) or epsilon variant, which was originally detected in California, carries spike glycoprotein mutations S13I in the signal peptide, $\mathrm{W} 152 \mathrm{C}$ in the N-terminal domain (NTD), and L452R in the receptorbinding domain (RBD). Plasma from individuals vaccinated with a Wuhan-1 isolate-based messenger RNA vaccine or from convalescent individuals exhibited neutralizing titers that were reduced 2- to 3.5fold against the B.1.427/B.1.429 variant relative to wild-type pseudoviruses. The L452R mutation reduced neutralizing activity in 14 of 34 RBD-specific monoclonal antibodies (mAbs). The S13I and W152C mutations resulted in total loss of neutralization for 10 of 10 NTD-specific mAbs because the NTD antigenic supersite was remodeled by a shift of the signal peptide cleavage site and the formation of a new disulfide bond, as revealed by mass spectrometry and structural studies ${ }^{4}$.

\section{TOXICITY OF THE SPIKE PROTEIN}

Available vaccines based on the Wuhan spike protein can be associated with rare, but serious side effects that must be monitored closely.

Serious side effects reported with Janssen COVID-19 vaccine are capillary leak syndrome, cerebral venous sinus thrombosis, deep venous thrombosis including pulmonary embolism, thrombocytopenia, transverse sinus thrombosis with thrombocytopenia, 
anaphylaxis, Guillain-Barré syndrome, seizures and vaccine-induced immune thrombotic thrombocytopenia. Similar side effects were also reported with AstraZeneca/Oxford COVID-19 vaccine (vaccine-induced immune thrombotic thrombocytopenia, deep venous thrombosis, capillary leak syndrome, cerebral venous sinus thrombosis). Serious side effects reported with mRNA Pfizer and Moderna vaccines are myocarditis, pericarditis and anaphylaxis 5 .

A case report of a deep vein thrombosis involving the right peroneal vein and extending up to the popliteal vein occurring shortly after the second dose of mRNA SARS-CoV-2 (BNT162b2, Comirnaty, Pfizer/BioNTech) vaccine was published by Carli and coworkers. Authors concluded that the intense immunological response evoked by the second dose of vaccine could be a trigger for the thrombotic event ${ }^{6}$.

Another case report of a 59-year-old woman presented to the Emergency Department with a 3-day history of sudden-onset left leg pain 7 days after receiving her first dose of BNT162b2 mRNA COVID-19 (PfizerBioNTech) was published. The patient was diagnosed with deep vein thrombosis (DVT) and pulmonary embolism (PE) and found to have a positive HIT screen with optical density (OD) of 0.6 via ELISA test. She was hospitalized for 4 days and discharged home with an oral anticoagulant (rivaroxaban). Authors concluded that this case report describes a possible link between BNT162b2 mRNA COVID-19 (Pfizer-BioNTech) vaccination and thromboembolism ${ }^{7}$.

SARS-CoV-2 infection relies on the binding of $\mathrm{S}$ protein (Spike glycoprotein) to $\mathrm{ACE}$ (angiotensin-converting enzyme) 2 in the host cells. Vascular endothelium can be infected by SARS-CoV-2, which triggers mitochondrial reactive oxygen species production and glycolytic shift. In a study, researchers showed that $\mathbf{S}$ protein alone can damage vascular endothelial cells (ECs) by downregulating ACE2 and consequently inhibiting mitochondrial function. A pseudovirus expressing $\mathrm{S}$ protein (PseuSpike) was administered to Syrian hamsters intratracheally. Lung damage was apparent in animals receiving Pseu-Spike, revealed by thickening of the alveolar septa and increased infiltration of mononuclear cells. Although the use of a noninfectious pseudovirus is a limitation to this study, the data of this study reveal that $\mathrm{S}$ protein alone can damage endothelium, manifested by impaired mitochondrial function and eNOS activity but increased glycolysis. It appears that $\mathrm{S}$ protein 
in ECs increases redox stress which may lead to AMPK deactivation, MDM2 upregulation, and ultimately ACE2 destabilization. It seems paradoxical that ACE2 reduction by S protein would decrease the virus infectivity, thereby protecting endothelium. However, a dysregulated renin-angiotensin system due to ACE2 reduction may exacerbate endothelial dysfunction, leading to endotheliitis ${ }^{8}$.

In another study researchers investigated the toxicity of spike protein in vitro using endothelial cell culture and recombinant SARS-CoV-2 spike protein S1 ReceptorBinding Domain (Spike). Mouse brain microvascular endothelial cells from normal (C57BL/6 mice) and diabetic ( $\mathrm{db} / \mathrm{db})$ mice were used. An endothelial transwell permeability assay revealed increased permeability in diabetic cells as well as after Spike treatment. The expression of VECadherin, an endothelial adherens junction protein, JAM-A, a tight junctional protein, Connexin-43, a gap junctional protein, and PECAM-1, were all decreased significantly after Spike treatment in control and to a greater extent, in diabetic cells. In control cells, Spike treatment increased association of endothelial junctional proteins with Rab5a, a mediator of the endocytic trafficking compartment. In cerebral arteries isolated from control and diabetic animals, Spike protein had a greater effect in downregulating expression of endothelial junctional proteins in arteries from diabetic animals than from control animals. Authors concluded that Spike-induced degradation of endothelial junctional proteins affects endothelial barrier function and is the likely cause of vascular damage observed in COVID-19 affected individuals ${ }^{9}$.

\section{MULTI-EPITOPE VACCINE}

All structural proteins of SARS-CoV-2 can be used as vaccine candidates. $\mathrm{N}$ protein is associated with replicase-transcriptase complexes and is involved with the packaging, transcription, and replication of the virus. $\mathrm{N}$ protein stimulates potent antibodies, which in turn may trigger cytokine production. Open reading frame $3 \mathrm{a}$ (ORF3a) is also essential for viral replication and virulence of SARS-CoV. ORF3a's function is to stimulate pro-IL-1 $\beta$ gene expression and IL-1 $\beta$ secretion that affect the lungs severely ${ }^{10}$.

A study has been published in the Journal of Biomolecular Structure and Dynamics which focuses on the evaluation of $\mathbf{E}, \mathbf{M}, \mathbf{N}$, ORF10, ORF8, ORF3a, and M proteins 
using bioinformatics tools to develop a multiepitope COVID-19 vaccine. In this study, bioinformatics approaches were employed to design and introduce a novel multi-epitope vaccine against 2019-nCoV that can potentially trigger both $\mathrm{CD}^{4+}$ and $\mathrm{CD}^{8+} \mathrm{T}$-cell immune responses and investigated its biological activities by computational tools. Three known antigenic proteins (Nucleocapsid, ORF3a, and Membrane protein, hereafter called NOM) from the virus were selected and analyzed for prediction of the potential immunogenic $\mathrm{B}$ and $\mathrm{T}$-cell epitopes and then validated using bioinformatics tools. Based on in silico analysis, the researchers have constructed a multi-epitope vaccine candidate (NOM) with five rich-epitopes domain including highly scored $\mathrm{T}$ and B-cell epitopes. After predicting and evaluating of the third structure of the protein candidate, the best $3 \mathrm{D}$ predicted model was applied for docking studies with Toll-like receptor 4 (TLR4) and HLA-A*11:01. In the next step, molecular dynamics (MD) simulation was used to evaluate the stability of the designed fusion protein with TLR4 and HLA-A*11:01 receptors. MD studies demonstrated that the NOM-TLR4 and NOM-HLA-A*11:01 docked models were stable during simulation time. In silico evaluation showed that the designed chimeric protein could simultaneously elicit humoral and cellmediated immune responses ${ }^{11}$.

\section{CONCLUSION}

Problems associated with Wuhan spike protein-based vaccines are the emergence of new SARS-CoV-2 strains that are more or less resistant to Wuhan spike protein-based vaccine-induced immunity, and toxicity of the spike protein (S protein alone can damage vascular endothelial cells).

All structural proteins of SARS-CoV-2 can be used as vaccine candidates (such as E, M, $\mathrm{N}$, ORF3a, and $\mathrm{M}$ proteins). Multi-epitope vaccine could recognize and assemble $B$ and T-cell epitopes that could efficiently trigger the immune system and elicit more potent immune response. Multi-epitope vaccine against SARS-CoV-2, excluding the spike protein, could potentially be less toxic than Wuhan spike protein-based vaccines, with less serious side effects, but still enough immunogenic to elicit protective immune response. Another potential benefit of a multi-epitope COVID-19 vaccine is that, taking into account the fact that most of the new SARS-CoV-2 strains carry significant 
new mutations in the spike protein and less significant mutations in other structural proteins of the virus, it could provide more potent immune response against new SARSCoV-2 variants. SARS-CoV-2 is fast enough to outsmart (by mutating in the spike protein) Wuhan spike protein-based vaccines, but would it be fast enough to outsmart multiepitope vaccine (by mutating in all structural proteins at the same time)?

\section{REFERENCES}

1. Edwards KM, Orenstein WA. COVID-19: Vaccines to prevent SARS-CoV-2 infection. Hirsch MS, Bloom A, ed. UpToDate. Waltham, MA: UpToDate Inc. https://www.uptodate.com (Accessed on August 22, 2021.)

2. Tracking SARS-CoV-2 variants. Available at https:/www.who.int/en/activities/trackingSARS-CoV-2-variants/ (Accessed on August 22, 2021)

3. Kannan SR, Spratt AN, Cohen AR, et al. Evolutionary analysis of the Delta and Delta Plus variants of the SARS-CoV-2 viruses. J Autoimmun. 2021;124: 102715.

4. McCallum M, Bassi J, Marco A, et al. SARSCoV-2 immune evasion by variant B.1.427/B.1.429. bioRxiv [Preprint]. 2021 Apr 1:2021.03.31.437925. doi: 10.1101/2021.03.31.437925.

5. Micromedex ${ }^{\circledR}$ (electronic version). IBM Watson Health, Greenwood Village, Colorado, USA. Available at: https://www.micromedexsolutions.com/ (cited: August/22/2021).

6. Carli G, Nichele I, Ruggeri M, et al. Deep vein thrombosis (DVT) occurring shortly after the second dose of mRNA SARS-CoV2 vaccine. Intern Emerg Med. 2021;16(3):803-804.

7. Al-Maqbali JS, Al Rasbi S, Kashoub MS, et al. A 59-Year-Old Woman with Extensive Deep Vein Thrombosis and Pulmonary Thromboembolism 7 Days Following a First Dose of the Pfizer-BioNTech BNT162b2 mRNA COVID-19 Vaccine. Am J Case Rep. 2021;22: e932946.

8. Yuyang L, Zhang J, Schiavon CR, et al. SARS-CoV-2 Spike Protein Impairs Endothelial Function via Downregulation of ACE 2. Circ. Res. 2021; 128:1323-1326.

9. Raghavan S, Kenchappa DB, Leo MD. SARS-CoV-2 Spike Protein Induced Degradation of Junctional Proteins That Maintain Endothelial Barrier Integrity. Front. Cardiovasc. 2021. https://doi.org/10.3389/fcvm.2021.687783

10. Bose P. A novel multi-epitope COVID-19 vaccine developed using reverse vaccinology. Available at https://www.newsmedical.net/news/20210531/A-novel-multiepitope-COVID-19-vaccine-developedusing-reverse-vaccinology.aspx (Accessed on August 22, 2021)

11. Enayatkhani M, Hasaniazad M, Faezi S, et al. Reverse vaccinology approach to design a novel multi-epitope vaccine candidate against COVID-19: an in-silico study. J Biomol Struct Dyn. 2020;39(8):2857-2872. 